\title{
Estudo Teórico da Relação Estrutura Atividade da Indolo [2, 1b] Quinazolina e seus Derivados Análogos Contra o Câncer de Ovário
}

\author{
Lilian T. F. M. Camargo, Hamilton B. Napolitano \& Ademir J. Camargo
}

The biological activities of the Indolo[2,1b]quinalozine and 33 analogue compounds were synthesized and tested against the human ovary cancer cells. The assays showed that 22 of these compounds exhibited cytotoxic activity. With the aim to correlate the physical chemical parameters of those compounds with the anticancer activity, the quantum molecular calculations were carried out in the compounds using the DFT theory. The Principal Component Analysis showed 4 electronic descriptors able to separate the compounds into two classes: active and inactive. The first component was responsible for the separation and describes $52 \%$ of the total variance of the original data.

Palavras-chave: Ovary cancer; structure activity relationship; indolo quinazoline.

The pharmaceutical industry is facing major challenges on the scientific and technological front of their Research \& Development (R\&D) programs. The modern research paradigm has been and continues to grow at a rapid pace, integrating knowledge of multi- and interdisciplinary teams. Recent advances in medicinal chemistry have created an important foundation in the search for new drug candidates. Modern drug design strategies employ computational methods such as molecular modeling as essential tools in the optimization of pharmacodynamic and pharmacokinetic properties of bioactive molecules. The aim of this chapter is to provide some fundamental concepts and applications of molecular modeling as a useful tool in the design of new drug candidates.

Keywords: molecular modeling; drug design; computational methods. 


\section{Introdução}

Câncer é o nome dado a um conjunto de mais de cem doenças que tem em comum o crescimento desordenado (maligno) de células que invadem os tecidos e órgãos, podendo espalhar-se (metástase) para várias regiões do corpo1. Conforme a Organização Mundial de Saúde (2007), ${ }^{1}$ o câncer tem sido responsável por cerca de 7,6 milhões $(13 \%)$ de todas as mortes ocorridas ao redor mundo. Sendo que $70 \%$ dos óbitos por câncer ocorreram em países de economia baixa ou média, devido às limitações no diagnóstico e tratamento da doença. No Brasil, estima-se que 190 mil pessoas morrem anualmente com algum tipo de câncer. A estimativa para 2020, em nível mundial, é de 10 milhões de morte por câncer ao ano e aproximadamente de 30 milhões de portadores da doença.

No mundo, milhares de mulheres são acometidos anualmente pelo câncer de ovário, representando um dos tipos de neoplasia com maior número de mortalidade entre pessoas do sexo feminino. ${ }^{2,3}$ Sendo o câncer ginecológico difícil de ser diagnosticado e o mais letal. Aproximadamente $75 \%$ dos tumores malignos de ovário são diagnosticados em estágios avançados embora seja menos freqüente que o câncer de colo do útero. ${ }^{4}$ As causas do câncer de ovário estão relacionadas com fatores ambientais, genéticos e hormonais. Cerca de $10 \%$ destes tipos de câncer são devidos a fatores genéticos e os outros $90 \%$ são esporádicos. ${ }^{4}$

Os tratamentos empregados no combate ao câncer não são eficazes e provocam muitos efeitos colaterais. Nas últimas décadas, o número de pesquisas visandoà descoberta de novos fármacos que sejam eficientes no tratamento desta doença tem crescido em todo mundo. Nesse sentido, um grupo de pesquisadores indianos isolaram do extrato da folha de Wrigthia tinctoria (uma planta medicinal indiana) a substância indolo[2,1b] quinazolina (Tryptanthrine). ${ }^{5}$ Esta molécula apresenta estrutura química muito similar à estrutura da batracyclin que é um potente composto contra o câncer melanoma, o que despertou nos pesquisadores interesse em sintetizá-la e realizar estudos biológicos.

Usando a indolo[2,1b]quinazoline como composto líder, uma série de derivados análogos foram sintetizados, os quais foram testados in vitro e in vivo contra o câncer de ovário, sendo que 24 destes compostos análogos mostraram- se ativos contra este tipo de câncer e 10 compostos foram considerados inativos nos testes biológicos. ${ }^{5} \mathrm{O}$ Presente trabalho investigou a relação entre as propriedades geométricas e eletrônicas das 34 moléculas e a sua atividade contra o câncer de ovário usando métodos de química quântica molecular para o cálculo dos descritores e métodos de estatística multivariada para discriminação dos compostos em ativos e inativos.

Inicialmente fez-se a análise conformacional dos compostos usando método semi-empírico PM36 implementado no software Hyperchem Release 7.5. ${ }^{7}$ A realização desta análise é importante, pois permite determinar a estrutura molecular que será utilizada no cálculo das propriedades eletrônicas e geométricas. Nem sempre a conformação de menor energia é a mais adequada para o estudo da atividade biológica, pois ao planejar um fármaco, procura-se a conformação que melhor ajuste ao receptor. Sendo assim, a escolha da conformação utilizada no cálculo dos descritores foi feita da seguinte forma: (1) selecionou-se a conformação mais estável da molécula mais ativa; (2) para os demais compostos foi selecionada a conformação cuja estrutura geométrica mais se assemelha à estrutura da conformação mais estável da molécula mais ativa. As geometrias das moléculas selecionadas foram otimizadas usando a Density Functional Theory (DFT) com o funcional de troca e correlação B3LYP ${ }^{8,9} \mathrm{e}$ o conjunto de funções de base 6-31G* implementados no pacote de programa Gaussian03. ${ }^{10}$ Após a otimização foram calculadas as propriedades moleculares (descritores) para os 34 compostos sob estudo. A Figura 1 mostra a estrutura química da indolo [2,1b] quinazolina com a respectiva numeração atômica adotada e seus derivados análogos.

Os descritores moleculares calculados neste trabalho com o objetivo de correlacioná-los com a atividade contra o câncer de ovário foram: $\log P$; volume $(V)$; polarizabilidade $(\alpha)$; ordens de ligação $\left(B_{n-m}\right.$ : ordem de liga) derivadas da análise populacional Natural Bond Order (NBO); ângulos de ligação $\left(A_{n_{-} m_{\_}}\right)$; ângulos diedrais $\left(D_{n_{-} m_{\_} p_{-}}\right)$; momento de dipolo $(\mu)$; energias dos orbitais de fronteira: Highest Occupied Molecular Orbital ( $\mathrm{E}_{\text {номо }}$ ) е Lowest Unoccupied Molecular Orbital ( $\left.\mathrm{E}_{\text {LUMO }}\right)$; dureza $(\eta)$; eletronegatividade de Mulliken $(\chi)$; Gap $\left(\mathrm{E}_{\text {номо }}-\mathrm{E}_{\text {LUмо }}\right)$ e cargas atômicas parciais $\left(c_{n}\right)$ derivada do potencial eletrostático usando o método ChelpG. ${ }^{11}$ 


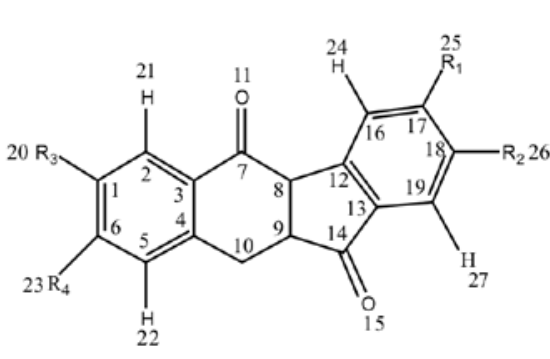

$$
\begin{aligned}
& 1 . \mathrm{R}_{1}=\mathrm{R}_{2}=\mathrm{R}_{3}=\mathrm{R}_{4}=\mathrm{H} \\
& \text { 2. } \mathrm{R}_{3}=\mathrm{Br} ; \mathrm{R}_{1}=\mathrm{R}_{2}=\mathrm{R}_{4}=\mathrm{H} \\
& \text { 3. } \mathrm{R}_{3}=\mathrm{NO}_{2} ; \mathrm{R}_{1}=\mathrm{R}_{2}=\mathrm{R}_{4}=\mathrm{H} \\
& \text { 4. } \mathrm{R}_{3}=\mathrm{R}_{4}=\mathrm{OMe} ; \mathrm{R}_{1}=\mathrm{R}_{2}=\mathrm{H} \\
& 5 . \mathrm{R}_{3}=\mathrm{NH}_{2} ; \mathrm{R}_{1}=\mathrm{R}_{2}=\mathrm{R}_{4}=\mathrm{H} \\
& \text { 6. } \mathrm{R}_{1}=\mathrm{BR} ; \mathrm{R}_{1}=\mathrm{R}_{2}=\mathrm{R}_{3}=\mathrm{H} \\
& \text { 7. } \mathrm{R}_{1}=\mathrm{NO}_{2} ; \mathrm{R}_{2}=\mathrm{R}_{3}=\mathrm{R}_{4}=\mathrm{H} \\
& 8 . \mathrm{R}_{1}=\mathrm{NH}_{2} ; \mathrm{R}_{1}=\mathrm{R}_{2}=\mathrm{R}_{3}=\mathrm{H} \\
& \text { 9. } \mathrm{R}_{1}=\mathrm{R}_{2}=-\mathrm{OCH}_{3}-\mathrm{R}_{3}=\mathrm{R}_{4}=\mathrm{H} \\
& 10 . \mathrm{R}_{1}=\mathrm{OMe}, \mathrm{R}_{2}=\mathrm{R}_{3}=\mathrm{R}_{4}=\mathrm{H} \\
& \text { 11. } \mathrm{R}_{1}=\mathrm{Cl} ; \mathrm{R}_{1}=\mathrm{R}_{2}=\mathrm{R}_{3}=\mathrm{H}
\end{aligned}
$$<smiles>[R]Nc1ccc2c(c1)C(=O)c1nc3ccccc3c(=O)n1-2</smiles>

12. $\mathrm{R}=\mathrm{C}(\mathrm{O}) \mathrm{CH}_{3}$ 13. $\mathrm{R}=\mathrm{C}(\mathrm{O}) \mathrm{CH}_{2} \mathrm{CH}_{2} \mathrm{CH}_{3}$ 14. $\mathrm{R}=\mathrm{C}(\mathrm{O}) \mathrm{CH}_{2} \mathrm{CH}_{3}$ 15. $\mathrm{R}=\mathrm{C}(\mathrm{O}) \mathrm{CH}_{2} \mathrm{Cl}$ 16. $\mathrm{R}=\mathrm{C}(\mathrm{O}) \mathrm{CH}_{2}$ 17. $\mathrm{R}=\mathrm{SO}_{2} \mathrm{CH}_{3}$<smiles>[R]C1(O)c2ccccc2-n2c1nc1ccccc1c2=O</smiles><smiles></smiles>

21. $R_{1}=R_{2}=R_{3}=R_{4}=R=H$

22. $\mathrm{R}_{1}=\mathrm{R}_{2}=\mathrm{R}_{3}=\mathrm{R}_{4}=\mathrm{H} ; \mathrm{R}=-\mathrm{CH}_{2} \mathrm{CH}_{2} \mathrm{OH}$

23. $\mathrm{R}_{1}=\mathrm{R}_{2}=\mathrm{R}_{3}=\mathrm{R}_{4}=\mathrm{H} ; \mathrm{R}=-\mathrm{CH}_{2} \mathrm{COOCH}_{3}$

24. $R_{1}=R_{2}=R_{3}=R_{4}=H ; R=-C H$<smiles>CCNc1ccccn1</smiles>

25. $\mathrm{R}_{1}=\mathrm{R}_{2}=\mathrm{R}_{3}=\mathrm{R}_{4}=\mathrm{H} ; \mathrm{R}=-\mathrm{C}(\mathrm{O}) \mathrm{CH}=\mathrm{CH}-\mathrm{Ph}$

26. $\mathrm{R}_{1}=\mathrm{R}_{2}=\mathrm{R}_{3}=\mathrm{R}_{4}=\mathrm{H} ; \mathrm{R}=-\mathrm{CH}_{2} \mathrm{CH}_{2} \mathrm{CH}_{2}-\mathrm{NMe}_{2}$ 27. $\mathrm{R}_{1}=\mathrm{R}_{2}=\mathrm{R}_{3}=\mathrm{R}_{4}=\mathrm{H} ; \mathrm{R}=-\mathrm{CH}_{2} \mathrm{CH}_{2} \mathrm{CH}_{2}-\mathrm{NMe}_{2}$

28. $\mathrm{R}_{1}=\mathrm{R}_{2}=\mathrm{R}_{3}=\mathrm{R}_{4}=\mathrm{R}=-\mathrm{CH}_{2}$

29. $\mathrm{R}_{1}=\mathrm{R}_{2}=\mathrm{R}_{3}=\mathrm{R}_{4}=\mathrm{H} ; \mathrm{R}=-\mathrm{CH}_{2} \backslash$

30. $\mathrm{R}_{1}=\mathrm{NO}_{2} ; \mathrm{R}_{2}=\mathrm{R}_{3}=\mathrm{R}_{4}=\mathrm{H} ; \mathrm{R}=-\mathrm{CH}_{2} \mathrm{CH}_{2}-\mathrm{NMe}_{2}$

$31 . \mathrm{R}_{1}=\mathrm{NO}_{2} ; \mathrm{R}_{2}=\mathrm{R}_{3}=\mathrm{R}_{4}=\mathrm{H} ; \mathrm{R}=-\mathrm{CH}_{2} \mathrm{CH}_{2} \mathrm{CH}_{2}-\mathrm{NMe}_{2}$

32. $\mathrm{R}_{1}=\mathrm{NO}_{2} ; \mathrm{R}_{2}=\mathrm{R}_{3}=\mathrm{R}_{4}=\mathrm{H} ; \mathrm{R}=-\mathrm{CH}_{2} \mathrm{CH}_{2} \mathrm{OH}$

33. $\mathrm{R}_{1}=\mathrm{Br} ; \mathrm{R}_{2}=\mathrm{R}_{3}=\mathrm{R}_{4}=\mathrm{H} ; \mathrm{R}=-\mathrm{CH}_{2} \mathrm{CH}_{2}-\mathrm{NMe}_{2}$

34. $\mathrm{R}_{1}=\mathrm{Br} ; \mathrm{R}_{2}=\mathrm{R}_{3}=\mathrm{R}_{4}=\mathrm{H} ; \mathrm{R}=-\mathrm{CH}_{2}$

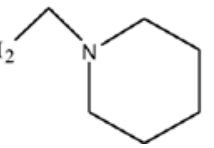

Figura 1: Estruturas moleculares e numeração atômica adotada nos cálculos da Indolo[2,1B] quinazoline e seus derivados análogos. 
As cargas derivadas do potencial eletrostático são ajustadas para reproduzir o potencial eletrostático ao redor da molécula. Muitos pesquisadores acreditam que a interação inicial entre o substrato e sítio ativo se dá, inicialmente, através de uma interação eletrostática. Nesse sentido, as cargas derivadas do potencial eletrostático constituem um importante descritor no estudo da relação estrutura atividade de moléculas bioativas.

As estruturas químicas e os valores de $\mathrm{GI}_{50}$ usados neste trabalho foram obtidos de Sharma et al. (2002). ${ }^{5}$ $\mathrm{O}$ parâmetro $\mathrm{GI}_{50}$ indica o potencial farmacológico (concentração do composto necessária para inibir 50\% das células cancerígenas) do composto. A Figura 1 ilustra os compostos, que podem ser divididos em dois grupos: ativos (6-8, 12-16 e 21-34), com $\mathrm{GI}_{50}$ até $100 \mu \mathrm{mol} / \mathrm{L}$, e inativos (1-5, 9-11 e 18-20), com GI ${ }_{50}$ acima de $100 \mu \mathrm{mol} / \mathrm{L}$.

A correlação entre os descritores calculados e a atividade dos derivados análogos contra o câncer de ovário foi feita usando métodos da estatística multivariada: peso de Fisher ${ }^{12}$ e Análise de Componentes Principais (PCA)13. $\mathrm{O}$ peso de Fisher é uma técnica que permite discriminar as variáveis mais significativas das variáveis menos significativas na classificação dos compostos. $\mathrm{O}$ peso de Fisher é calculado usando Equação 1

$$
W_{A B}(i)=\frac{\left[\bar{x}_{i}(A)-\bar{x}_{i}(B)\right]^{2}}{S_{i}^{2}(A)+S_{i}^{2}(B)}
$$

onde A e B são as classes consideradas na investigação, $\mathrm{i}$ representa as variáveis ou descritores, $\bar{x}_{i}(A)$ é a média do descritor i na classe $\mathrm{A}, \bar{x}_{i}(B)$ é a média do descritor i na classe B. $S_{i}^{2}(A)$ é a variância do descritor $i$ na classe A e $S_{i}^{2}(B)$ é a variância do descritor $i$ na classe B. Observa-se que quanto maior a diferença entre as médias das classes $\mathrm{A}$ e B e quanto menor as variâncias dentro das classes, maior é o valor do peso de Fisher. Como variâncias menores são melhores para discriminação das classes, quanto maior o peso de Fisher maior a capacidade da variável na separação. ${ }^{12}$

A Análise de Componentes Principais (PCA) consiste em calcular novas variáveis como combinação linear das variáveis antigas de tal modo que as novas variáveis ou componentes sejam ortogonais entre si. Trata-se de um método não supervisionado da estatística multivariada. ${ }^{13}$ As componentes principais conservam a variância das variáveis originais e como são ortogonais não existe correlação entre elas. Esta característica das componentes principais é importante, pois, garante que cada componente descreva uma "característica" diferente dos dados originais. ${ }^{14}$

Do ponto de vista matemático, a PCAé um método que consiste em decompor uma matriz de dados originais $\mathbf{X}$ de posto $\mathrm{r}$ em uma soma de $\mathbf{r}$ matrizes de posto 1 , em que o posto é o número de vetores linearmente independentes da matriz X. As matrizes de posto 1 são os produtos dos vetores de scores $\left(S_{\mathrm{h}}\right)$ pelos transpostos dos vetores de loadings $\left(l_{\mathrm{h}}\right)$, como mostra a Equação 2.14,15

$$
\mathrm{X}=\mathrm{S}_{1} \cdot l_{1}^{T}+\mathrm{S}_{2} \cdot l_{2}^{T} \cdots+\mathrm{S}_{h} \cdot l_{h}^{T}
$$

A principal vantagem no uso da análise de componentes principais é a possibilidade de diminuir a dimensão dos dados originais. Em geral, umas poucas componentes são suficientes para descrever as características de interesse de um conjunto de dados. Um conjunto formado por $m$ variáveis e $k$ amostra, representa $k$ pontos em um espaço de $m$ dimensões. Se $m$ for maior que três não é possível a visualização gráfica. As primeiras PCs, isto é, 2 ou 3, na maioria das vezes, são capazes de explicar a maior parte da variância dos dados originais. $\mathrm{O}$ espaço $\mathrm{m}$ dimensional se reduz para aproximadamente 3 dimensões, tornando possível a análise gráfica dos dados. ${ }^{12-15}$

Para ter uma visão preliminar de quais descritores seriam mais relevantes na separação das classes ativas e inativas, o peso de Fisher foi calculado para os 80 descritores obtidos dos cálculos quanto-mecânicos para os 34 compostos sob estudo. Os valores dos pesos de Fisher calculados variaram de 0,00 a 2,05. A partir da análise dos pesos de Fisher, foram considerados para análise de componentes principais somente as variáveis com peso de Fisher maior do que 0,20. As variáveis com peso de Fisher maior do que 0,20 são mostradas na Tabela 1 .

Como as variáveis apresentam unidades diferentes é necessário autoescalar os dados para que todas as variáveis possam ter a mesma importância na análise de componentes principais. No autoescalamento cada variável é escalada para ter média zero e variância unitária.16 Usando os descritores (variáveis) selecionados pelo peso de Fisher (Tabela 1), após inúmeras tentativas, a melhor separação foi obtida usando as variáveis B12_13, B19_27, B9_14, B5_22 (onde B representa ordem de ligação). Os valores destas variáveis são mostrados na Tabela 2 . 
Tabela 1: Descritores selecionados com peso de Fischer maior que 0,20

\begin{tabular}{|c|c|c|c|}
\hline Descritor & Peso de Fisher & Variáveis & Peso de Fisher \\
\hline$C_{14}$ & 0,89 & $\mathrm{~B}_{12 \_13}$ & 2,05 \\
\hline$c_{l 5}$ & 1,02 & $\mathrm{~B}_{13 \_14}$ & 0,88 \\
\hline$c_{l 9}$ & 0,28 & $\mathrm{~B}_{14 \_15}$ & 0,27 \\
\hline$c_{27}$ & 0,40 & $\mathrm{~B}_{17.25}$ & 0,24 \\
\hline$c_{34}$ & 0,75 & $\mathrm{~B}_{19 \_27}$ & 0,21 \\
\hline $\mathrm{B}_{4110}$ & 0,68 & V & 0,48 \\
\hline $\mathrm{B}_{5 \_22}$ & 0,33 & GAP & 0,39 \\
\hline $\mathrm{B}_{5-6}$ & 0,20 & $\eta$ & 0,39 \\
\hline $\mathrm{B}_{8 \_9}$ & 0,83 & $\mathrm{~A}_{3-7,11}$ & 0,23 \\
\hline $\mathrm{B}_{8 \_12}$ & 0,39 & $\mathrm{~A}_{11}{ }_{2}{ }_{2} 10$ & 0,30 \\
\hline $\mathrm{B}_{9,14}$ & 1,83 & $A_{13 \_14 \_15}$ & 0,77 \\
\hline
\end{tabular}

Tabela 2: Valores das ordens de ligação responsáveis pela discriminação das classes a nível de teoria B3LYP/6-31G*

\begin{tabular}{|l|l|l|l|}
\hline $\mathbf{B}_{\mathbf{5} \mathbf{2} 2}$ & $\mathbf{B}_{\mathbf{9}} \mathbf{1 4}$ & $\mathbf{B}_{\mathbf{1 2} \mathbf{1} \mathbf{1 3}}$ & $\mathbf{B}_{\mathbf{1 9} \mathbf{2} \mathbf{}}$ \\
\hline 1,4803 & 1,3033 & 1,4023 & 0,9123 \\
1,4825 & 1,3040 & 1,4038 & 0,9022 \\
1,4909 & 1,3034 & 1,4074 & 0,8891 \\
1,4278 & 1,3033 & $1, \mathbf{4 0 1 6}$ & 1,0282 \\
1,5178 & 1,3048 & 1,3993 & 0,9141 \\
1,4792 & 1,3053 & 1,3932 & 0,9121 \\
1,4763 & 1,2943 & 1,3836 & 0,9110 \\
1,4827 & 1,3023 & 1,3871 & 0,9129 \\
1,4794 & 1,3232 & 1,3715 & 0,9125 \\
1,4811 & 1,3214 & 1,3727 & 0,9126 \\
1,4809 & 1,3089 & 1,3917 & 0,9125 \\
1,4800 & 1,3068 & 1,3845 & 0,9120 \\
1,4800 & 1,3058 & 1,3867 & 0,9120 \\
1,4803 & 1,3002 & 1,3932 & 0,9120 \\
1,4804 & 1,2995 & 1,3917 & 0,9122 \\
1,4814 & 1,2983 & 1,3941 & 0,9127 \\
1,4804 & 1,3077 & 1,3843 & 0,9121 \\
1,4870 & 1,3328 & 1,3955 & 0,9141 \\
1,4860 & 1,3391 & 1,3942 & 0,9138 \\
1,4852 & 1,3305 & 1,3961 & 0,9135 \\
1,4892 & 1,2966 & 1,4032 & 0,9137 \\
1,4876 & 1,2937 & 1,4044 & 0,9132 \\
1,4889 & 1,2960 & 1,4031 & 0,9136 \\
1,4890 & 1,2962 & 1,4034 & 0,9138 \\
1,4870 & 1,2946 & 1,4052 & 0,9133 \\
1,4901 & 1,2960 & 1,4032 & 0,9140 \\
1,4901 & 1,2957 & 1,4028 & 0,9139 \\
1,4902 & 1,2959 & 1,4032 & 0,9140 \\
1,4906 & 1,2962 & 1,4030 & 0,9141 \\
1,4860 & 1,2824 & 1,3856 & 0,9127 \\
1,4861 & 1,2828 & 1,3954 & 0,9128 \\
1,4836 & 1,2813 & 1,3874 & 0,9120 \\
1,4887 & 1,2946 & 1,3972 & 0,9136 \\
1,4887 & 1,2946 & 1,3972 & 0,9136 \\
\hline
\end{tabular}

A Figura 2 mostra que a primeira componente (PC1) é responsável pela discriminação entre compostos ativos (1, 5-8, 12-16 e 21-34) e inativos (2-4, 9-11 e 17-20). Sozinha, esta componente explica $52,56 \%$ da variância total dos dados. As duas primeiras componentes explicam juntas $79,01 \%$. Considerando as três primeiras componentes, $94,42 \%$ da variância dos dados são explicadas. A Figura 3 mostra os loadings (pesos) das variáveis responsáveis pela separação.

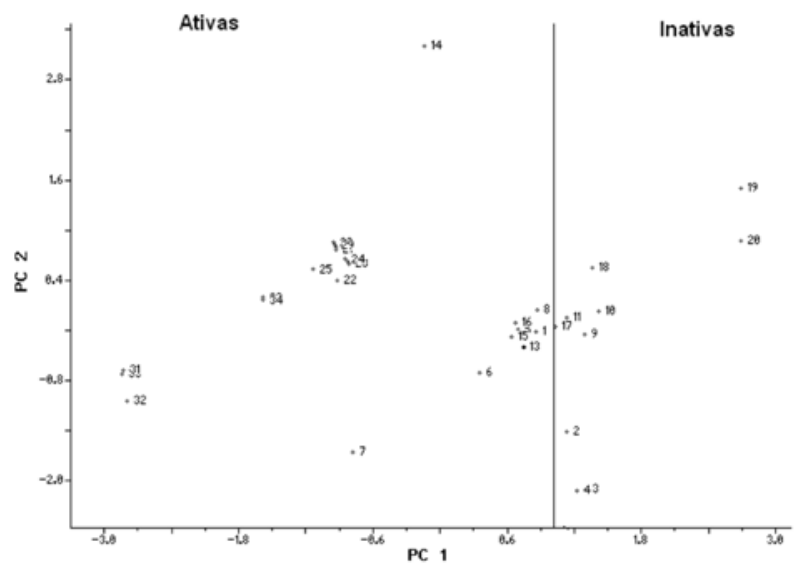

Figura 2: Representação gráfica dos scores obtidos usando o programa Einsight 3.0.

A Equação 3 mostra a influência dos loadings no cálculo dos scores da PC1, a qual foi responsável pela discriminação das classes em ativa e inativa.

$P C l=-0,1915 b_{5.22}-0,6114 b_{9.14}+0,6075 b_{12.13}+0,4695 b_{19.27} \quad$ Eq. (3)

A ordem de ligação mostra a densidade eletrônica entre átomos ligados. Quanto maior a ordem de ligação maior a energia de dissociação e menor a distância de ligação. A Equação 3 e a Figura 3 mostram que para um composto pertencer ao grupo dos compostos ativos deve apresenta altos valores numéricos das variáveis $\left(\mathrm{B}_{9{ }_{14}} \mathrm{e}\right.$ B5_22) e baixos valores numéricos das variáveis $\left(B_{12 \_13}\right.$ e $\mathrm{B}_{1927}$ ). Analisando os dados da Tabela 3, observa-se que a ordem de ligação com maior peso na separação das variáveis foi a ordem de ligação entre os átomos 9 e 14 (veja Figura 1) e a variável de menor peso na primeira componente foi a ordem de ligação entre os átomos 5 e 22 , sendo esta variável a de maior peso para segunda componente. 


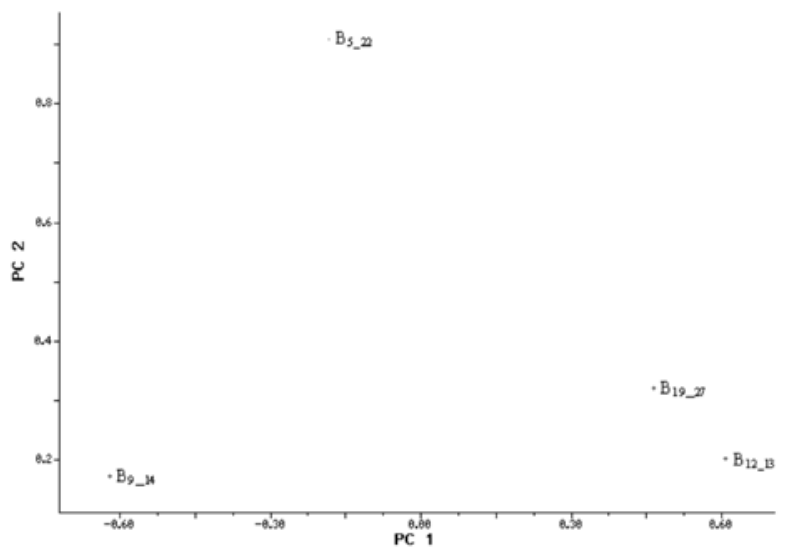

Figura 3: Loadings (pesos) das variáveis na primeira e segunda componente

Não é uma tarefa simples prever a interação entre uma droga e seu receptor biomolecular quando não se conhece a estrutura molecular do sítio receptor alvo. Contudo, com as informações até aqui obtidas observa-se que as variáveis responsáveis pela separação das moléculas, entre ativas e inativas, são todas propriedades eletrônicas. Isto nos permite postular que os efeitos eletrônicos têm importância significativa no entendimento das atividades das indolos contra o câncer de ovário e, possivelmente, a interação entre a droga e o receptor ocorre através da transferência de cargas ou por formação de ligação.

Tabela 3: Loadings (pesos) das variáveis na primeira e segunda componente

\begin{tabular}{|c|c|c|}
\hline Variável & PC1 & PC2 \\
\hline$B_{5 \_22}$ & $-0,18$ & 0,91 \\
$B_{9, / 4}$ & $-0,62$ & 0,17 \\
$B_{12 / 13}$ & 0,61 & 0,20 \\
$B_{I 927}$ & 0,46 & 0,32 \\
\hline
\end{tabular}

Onde $\mathrm{B}_{n \_m}$ representa a ordem de ligação entre o átomo $n$ e $m$.

\section{CONCLUSÃO}

Usando a Análise de Componentes Principais (PCA), foi possível identificar os descritores calculados teoricamente que estão correlacionados com a atividade contra o câncer de ovário. Os descritores que melhor discriminaram as moléculas em duas classes (ativas e inativas) foram as ordens de ligação entre os átomos (Figura 1): 5-22, 9-14, 12-13 e 19-27. Com base nos resultados, é possível mapear indicativos para a síntese novos compostos que sejam ativos contra o câncer de ovário, ou seja, é necessário aumentar o valor numérico das variáveis $\left(\mathrm{B}_{5 \_22}\right.$ e $\left.\mathrm{B}_{9 \_14}\right)$ e diminuir o valor numérico das variáveis $\left(\mathrm{B}_{12 \_13}\right.$ e $\left.\mathrm{B}_{1927}\right)$ que foram significativas na separação das moléculas quanto à sua atividade.

Cabe destacar que no processo de descoberta de um novo fármaco ${ }^{18,19}$ os testes experimentais norteiam os estudos teóricos, que melhoram o entendimento sobre o potencial fármaco, que propõe novas moléculas que sejam potencialmente mais eficientes, que devem ser testadas experimentalmente. Trata-se, portanto, de um processo fundamentalmente iterativo. ${ }^{19}$

\section{AGRADECIMENTOS}

Os autores agradecem à Pró-Reitoria de Pesquisa e Pós-Graduação da Universidade Estadual de Goiás, e à Coordenação de Aperfeiçoamento de Pessoal de Nível Superior (processo 588605/2007), pelo suporte a realização deste trabalho.

\section{REFERÊNCIAS}

1. World Health Organization. New guide on palliative care services for people living with advanced cancer, $51 \mathrm{p}, 2007$.

2. http://www.inca.org.br/cancer, acessada em janeiro de 2008.

3. Paulinelli, R. R.; Freitas Junior, R., Moreira. A.R.; Rev. Bras. Mast. 2003, 3, 168.

4. Temmink O. H.; Prins H. J.; van Gelderop E.; Peters G. J.; British journal of cancer. 2007, 96, 61 .

5. Sharma V. M.; Prasanna, P.; Seshu, K. V. A.; Renuka, B.; Rao, C. V. L.; Kumar, G. S.; Narasimhulu, C. P.; Babu, P. A.; Puranik, R. C.; Subramanyam, D.; Venkateswarlu, A.; Rajagopal, S.; Kumar, K. B. S.; Rao, C. S.; Mamidi, N. V. S. R.; Deevi, D. S.; Ajaykumar, R.; Rajagopalan, R.; Bioorganic \& Medicinal Chemistry. 2002, 12, 2303.

6. Stewart, J. J. P.; J. Comp. Chem. 1989, 10, 209; Stewart, J. J. P.; J. Comp. Chem. 1989, 10, 221.

7. Hyperchem, Release 7.0, Hypercube Inc. $1115 \mathrm{NW}$ 4th Street, Gainesville, FL 32601, USA.

8. LEE, C.; YANG, W.; PARR, R. G.; Phys. Rev. 1988, B37, 785 . 


\section{Artigo 1}

9. BECKE, A. D.; Phys. Rev. 1988, A38, 3098.

10. Frisch, M. J.; Trucks, G. W.; Schlegel, H. B.; Scuseria, G. E.; Robb, M. A.; Cheeseman, J. R., J. A. Montgomery, JR.; Vreven T.; Kudin K. N.; Burant, J. C.; Millam, J. M.; Iyengar S. S.; Tomasi J.; Barone, V.; Mennucci, B.; Cossi, M.; Scalmani, G.; Rega, N.; Petersson G. A.; Nakatsuji, H.; Hada, M.; Ehara, Toyota, M., K.; Fukuda, R.; Hasegawa, J.; Ishida, M.; Nakajima, T.; Honda, Y.; Kitao, O.; Nakai, H.; Klene, M., Li, X.; Knox, J. E.; Hratchian, H. P.; Cross, J. B.; Adamo, C., Jaramillo, J.; Gomperts R.; Stratmann, R. E.; Yazyev, O.; Austin, A. J.; Cammi, R.; Pomelli, C.; Ochterski, J. W.; Ayala, P. Y.; Morokuma K.; Voth, G. A.; Salvador, P.; Dannenberg, J. J., Zakrzewski, V. G.; Dapprich, S.; Daniels, A. D.; Strai,N M. C.; Farkas, O.; Malick, D. K.; Rabuck, A. D.; Raghavachari, K.; Foresman, J. B.; Ortiz, J. V.; Q. CUI, Baboul, A. G.; Clifford, S.; Cioslowski, J.; Stefanov, B. B.; Liashenko, G. LIU, A.; Piskorz P., Komaromi, I.; Martin R. L.; Fox, D. J.; Keith, Al-laham, T. M. A.; Peng, C. Y.; Nanayakkara, A.; Challacombe, M.; Gill, P. M. W.; Johnson, B.; Chen, Wong, W.; Gonzalez, M. W.; C.; Pople, J. A.; Gaussian 03, Revision B.04, gaussian, inc., Pittsburgh pa, 2003.

11. Breneman, C. M.; Wiberg, K. B.; J. Comp. Chem. 1990, 11,361 .

12. Sharaf, M.A.; Illman, D.L.; Kolwalski, B. R. Chemometrics, JonhWiley, New York, 1986.

13. Press, S.J. Applied multivariate statistics. New York, Holts, Rinehart and Winston, 1972.

14. Massart, D. L; Chemometrics tutorial, Elsevier:
Amsterdam, 1990.

15. Magalhães, D.; Bruns, R. E.; Vasconcellos, P. C.; Quim. Nova, 2007, 30, 577.

16. Camargo, J. C.; Dissertação de mestrado. Instituto de Química de São Carlos, Universidade de São Paulo, 1998.

17. Neto, B. B.; Scarminio, I. S.; Bruns, R. E.; Planejamento e otimização de experimentos, UNICAMP: Campinas, 1995.

18. Barreiro, E. J.; Fraga, C. A. M.; Química medicinal: as bases moleculares da ação dos fármacos. Artmed Editora: Porto Alegre, 2001.

19. Thomas, G.; Fundamentals of medicinal chemistry, Wiley:West Sussex, 2003.

\section{Lilian T. F. M. Camargo, Hamilton B. Napolitano \& Ademir J. Camargo*}

\footnotetext{
${ }^{1}$ Unidade Universitária de Ciências Exatas e Tecnológica, Universidade Estadual de Goiás, Campus Henrique Santillo, BR 153, Km 98 - CEP 75001-970 Fone: (62) 3328-1156 Anápolis-GO, Brasil.
}

*E-mail: ajc@ueg.br 